\title{
Isolation of anti-CD22 Fv with high affinity by Fv display on human cells
}

\author{
Mitchell Ho, Satoshi Nagata, and Ira Pastan* \\ Laboratory of Molecular Biology, Center for Cancer Research, National Cancer Institute, National Institutes of Health, Bethesda, MD 20892-4264 \\ Contributed by Ira Pastan, May 4, 2006
}

In vitro antibody affinity maturation has generally been achieved by display of mouse or human antibodies on the surface of microorganisms (phage, bacteria, and yeast). However, problems with protein folding, posttranslational modification, and codon usage still limit the number of improved antibodies that can be obtained. An ideal system would select and improve antibodies in a mammalian cell environment where they are naturally made. Here we show that human embryonic kidney 293T cells that are widely used for transient protein expression can be used for cell surface display of single-chain Fv antibodies for affinity maturation. In a proof-of-concept experiment, cells expressing a rare mutant antibody with higher affinity were enriched 240 -fold by a single-pass cell sorting from a large excess of cells expressing WT antibody with a slightly lower affinity. Furthermore, we successfully obtained a highly enriched mutant with increased binding affinity for CD22 after a single selection of a combinatory library randomizing an intrinsic antibody hotspot. Important features are that one display selection cycle requires only 1 week, and transfection of cells in a single $100-\mathrm{mm}$ dish produces $10^{7}$ individual clones so that a repertoire of $10^{9}$ is feasible under current experimental conditions.

antibody engineering | B cell malignancy | FACS | human embryonic kidney cells | receptors

reating high-affinity antibodies to important biomolecules for clinical use is an important and challenging task. Seventeen therapeutic mAbs have been approved by the Food and Drug Administration, and many more mAbs will be approved in the next few years (reviewed in ref. 1). The levels of antibody affinity obtained from hybridomas are often not sufficient for effective clinical use in part because of the in vivo affinity ceiling $(2,3)$; therefore an improvement of antibody affinity is often required (4). For the past 2 decades phage display was used for in vitro antibody affinity maturation (5-10), and more recently, bacterial (11-13) and yeast cell surface display systems have been developed (14-16). A significant advantage of cell surface display over phage display is the use of quantitative flow cytometric sorting and analysis to identify high-affinity interactions and normalize for antibody protein expression. Cell-free systems like ribosome display are useful tools in antibody affinity maturation because of the large size of libraries $\left(10^{13}\right.$ to $\left.10^{14}\right)$ that can be produced and analyzed (17-19). These techniques have all been successful, but problems with protein folding, posttranslational modification, and codon usage limit the number of improved antibodies that can be obtained (20). Many potential therapeutic proteins are highly aggregation-prone upon heterologous expression in bacteria (21). Moreover, serious translational problems can occur when proteins selected in bacteria, yeast, or cell-free systems are transferred into mammalian expression systems to produce therapeutic antibodies.

Mammalian cells such as Chinese hamster ovary cells are the dominant system used for the production of the majority of therapeutic antibodies in the pharmaceutical industry (22). Therefore, mammalian cells should be used in antibody selection to avoid problems caused by selection in microorganisms. To use an animal cell environment for antibody selection and affinity maturation, a hypermutating chicken B lymphoma line (23) and a retrovirus display system (20) have been developed. Recently, a random peptide library fused to CCR5 and expressed on the mammalian cell surface has been reported (24). The potential of this display system has been demonstrated for small peptides of 9 or 13 aa. A mammalian cell surface-displayed antibody system, which is robust and comparable to existing phage and yeast display technologies used in antibody engineering, has not yet been reported. Here, we show that human cells can be engineered for cell surface display of functional single-chain Fv $(\mathrm{scFv})$ antibodies and is suitable for affinity maturation. For this purpose we have used the anti-CD22 $\mathrm{scFv}$ fused to the transmembrane domain of human platelet-derived growth factor receptor (PDGFR) displayed on human embryonic kidney (HEK) $293 \mathrm{~T}$ cells. We find that the mammalian cell scaffold allows a 240-fold single-pass enrichment of a rare higher-affinity mutant from a large excess of only slightly lower-affinity WT antibody. Furthermore, we show that a mutant with an increased binding affinity for CD22 can be obtained after a single-pass selection of a combinatory library randomizing an intrinsic antibody hotspot.

\section{Results}

Expression and Surface Localization of scFv. We first determined whether HEK 293T cells, widely used for transient protein expression, could be engineered for cell surface display of functional antibody Fvs. Because of our interest in targeting CD22-expressing cancers, we chose the anti-CD22 scFv RFB4 fused to the transmembrane domain of PDGFR as the experimental system. The expression vector contains the cytomegalovirus promoter and the nucleotide sequence encoding the murine Ig $\kappa$ chain signal peptide (METDTLLLWVLLLWVPGSTGDJ) and the transmembrane domain (amino acids 514-562) of PDGFR (25). Fusion to the N-terminal portion of PDGFR has been used to anchor other proteins such as a virus hemagglutinin (26), toll-like receptors (27), and a chimeric human IgG-cat allergen fusion protein (28).

We expressed WT anti-CD22 BL22 (RFB4) scFv or its high-affinity derivative, HA22 scFv, on HEK 293 T human cells (Fig. 1A). For comparisons, scFvs were also expressed in phage (10) or yeast display systems. Fig. $1 B$ shows a structural model of the BL22 Fv. The protein contains a myc epitope tag at the carboxyl terminal of the $\mathrm{scFv}$ that is used to measure the expression level. A time-course experiment indicated that the expression level of transfected scFv-PDGFR is maximized 48-72 $\mathrm{h}$ after transfection (data not shown). Surface localization of the scFv-PDGFR fusion was verified by confocal fluorescence microscopy and flow cytometry. Cells labeled simultaneously with biotinylated CD22-Fc proteins and an anti-c-myc mAb were examined by laser scanning confocal microscopy (Fig. 2). Cells bearing the surface display vector expressing the scFv-PDGFR

Conflict of interest statement: No conflicts declared.

Abbreviations: $\mathrm{CDR}$, complementary determining region; HEK, human embryonic kidney; $\mathrm{MFI}$, mean fluorescence intensity; $\mathrm{PE}$, phycoerythrin; PDGFR, platelet-derived growth factor receptor; PT, Pro-91-Thr-92; scFv, single-chain Fv.

*To whom correspondence should be addressed. E-mail: pastani@mail.nih.gov. 
A

\begin{tabular}{c|c|c|c|c|c|c|c|c|}
\hline Mammalian & SP & VH & L & \multicolumn{2}{|c|}{ VL } & T & \multicolumn{2}{c|}{ PDGFR } \\
\cline { 2 - 8 } Phage & SP & VH & L & VL & T & g3p \\
neast & SP & Aga2 & VH & L & VL & T \\
\cline { 2 - 8 }
\end{tabular}

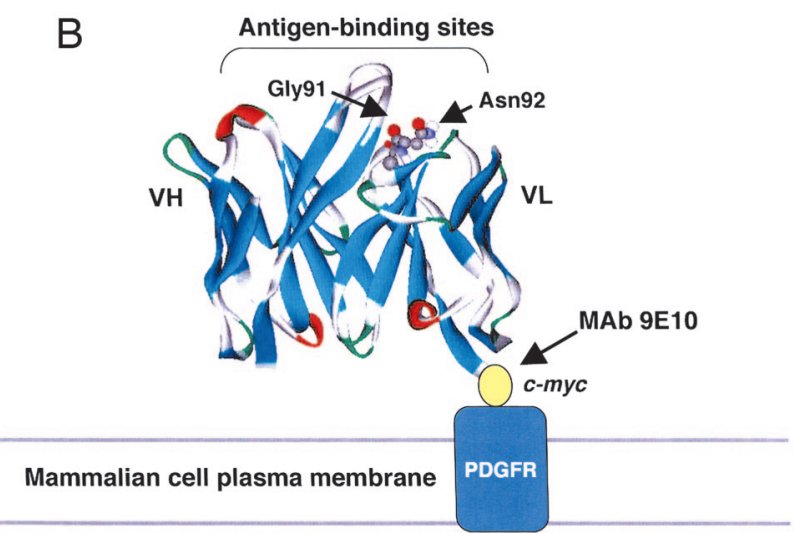

Fig. 1. Mammalian cell surface display. (A) Cell surface display of scFv on mammalian cells and comparison with other display systems. In the mammalian (HEK 293T) cell surface display system, the scFv (anti-CD22) containing $\mathrm{VH}$-linker (L)-VL is fused to the N-terminal portion of the PDGFR transmembrane domain. Murine Ig $\kappa$-chain signal peptide (SP) and c-myc epitope tag (T) are used (see Materials and Methods). In a M13 phage display system, the scFv with the g3p signal peptide is fused to the N-terminal of g3p (10). In a typical yeast cell surface display system, the scFv with A-agglutinin signal peptide fused to the $\mathrm{C}$-terminal portion of $\mathrm{S}$. cerevisiae a-agglutinin yeast adhesion receptor (Aga2) (see Materials and Methods). $\mathrm{VH}$, Ig heavy-chain variable region; VL, Ig light-chain variable region. (B) Schematic illustration of surface display on mammalian cells. An additional 10-aa epitope tag (c-myc) was fused to the $C$ terminus of the scFv (based on the anti-CD22 RFB4 Fv structural model) (10), allowing quantitation of fusion display with mAb $9 E 10$ independent of antigen (CD22) binding. Fusion to the $\mathrm{N}$-terminal portion of the PDGFR transmembrane domain was used to anchor scFv on the mammalian (HEK 293T) cell surface. In the present study, Gly-91 and Asn-92 located in VL are mutagenized for affinity maturation.

fusion protein were colabeled (yellow) by a mixture of the CD22-Fc (red) and the anti-c-myc antibody (green) (Fig. 2). In contrast, control HEK $293 \mathrm{~T}$ cells were not labeled by CD22-Fc or anti-c-myc mAb (data not shown).

FACS staining of the scFv-PDGFR fusion protein on the cell surface $48 \mathrm{~h}$ after transfection is shown in Fig. 3. The scFvs were reacted with biotinylated $\mathrm{CD} 22-\mathrm{Fc}$ proteins to assess CD22specific binding and simultaneously with an anti-c-myc mAb 9E10 to normalize expression levels of the scFv. Two-color flow cytometric analysis of the HEK 293T cells demonstrated both scFv expression (FITC for the level of 9E10 binding) and specific binding to the CD22 antigen [phycoerythrin (PE) level for CD22 binding]. The diagonal-like expression profile in Fig. $3 B$ indicates that the transfected HEK 293 T cells express various numbers of scFvs on the cell surface, each of which can bind to CD22. CD22 binding was not prevented by adding a CD30-Fc protein (data not shown), indicating that $\mathrm{scFv}$ binding to $\mathrm{CD} 22$ is specific. Likewise, when an isotype control mAb was used instead of 9E10, no antigen expression (FITC) was detected (data not shown), indicating that the FITC staining is caused by a specific interaction between the c-myc epitope tag and 9E10. These results demonstrate that mammalian HEK $293 \mathrm{~T}$ cells can be engineered to display a functional antibody Fv at the cell surface. Moreover, antigen binding and Fv expression can be measured
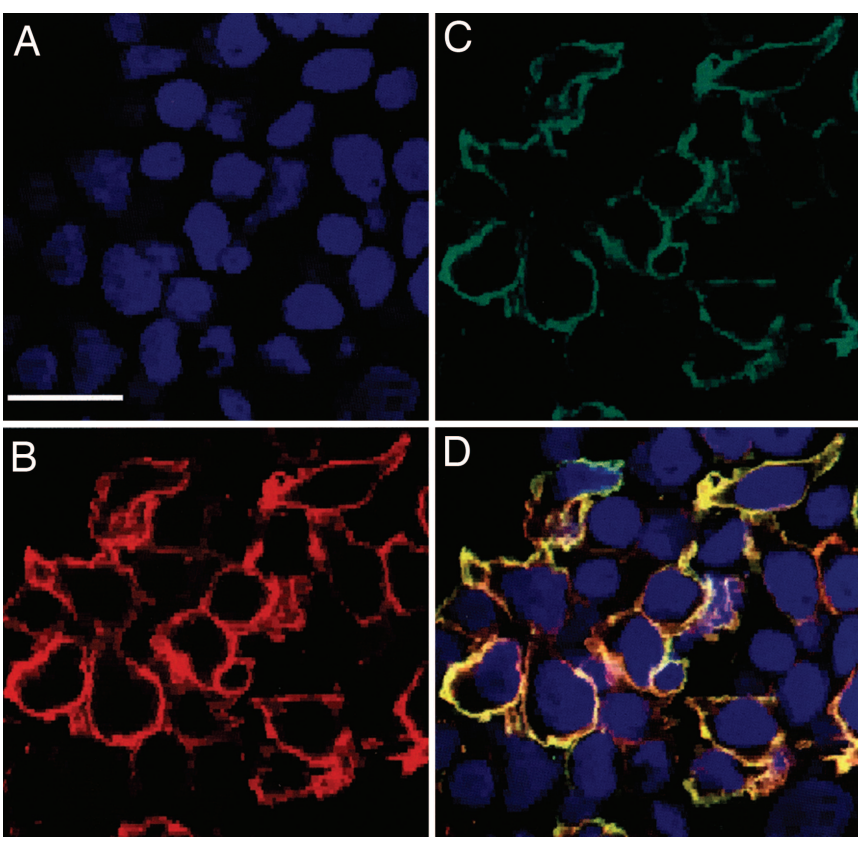

Fig. 2. Confocal microscopic images of HEK $293 \mathrm{~T}$ cells displaying scFv. HEK 293T cells transfected with a plasmid directing surface expression of anti-CD22 scFv were grown on coverslips. $(A-C)$ Transfected cells were fixed with DAPI nuclear staining $(A)$ followed by detection with biotinylated $C D 22-\mathrm{Fc}(B)$, and anti-c-myc antibody (C) followed by Steptavidin-Alexa Fluor-594 (red) and anti-mouse IgG-Alexa Fluor-488 (green). (D) Merged staining patterns (yellow) are shown. (Scale bar: $25 \mu \mathrm{m}$.)

simultaneously and quantitatively, an important feature in obtaining Fvs with improved affinities.

The same anti-CD22 scFv was displayed on the surface of yeast (Fig. $3 C$ and $D$ ). We found $30-40 \%$ of yeast cells did not express $\mathrm{scFv}$ on their surface, resulting in two histogram peaks. The presence of nonexpressing population is consistent with published yeast display experiments $(14,16)$.

Fine Discrimination by Mammalian Cell Surface Display. To determine whether this system could discriminate between Fvs with different affinities, a step essential in affinity maturation, we chose the BL22 scFv, now in clinical use, and a 2-fold higheraffinity mutant (HA22) and displayed them on the surface of HEK 293T cells. The scFv BL22-displaying cells were mixed with scFv HA22-displaying cells at a 400:1 ratio. This ratio was chosen because in a typical hotspot-based selection, DNA encoding two amino acids is mutagenized and the library has $(20 \times 20) 400$ mutants with different amino acids. The cell mixture was incubated in $1 \mathrm{nM} \mathrm{CD} 22-\mathrm{Fc}$ and $9 \mathrm{E} 10$ at $25^{\circ} \mathrm{C}$ for $1 \mathrm{~h}$. Cells were then fluorescently labeled and sorted. The flow cytometric sorting instrument used here can sort 10,000-20,000 cells per s. Sorting of $10^{7}$ cells (one $100-\mathrm{mm}$ dish) takes $\approx 10 \mathrm{~min}$. The sort window was set to obtain the top $0.1 \%\left(\approx 10^{4}\right)$ high CD22 binders $(29)$ (Fig. 4). The window covers an area with a range of different scFv expression levels measured by the anti-c-myc antibody so that mutant scFvs with a high affinity to CD22 can be selected regardless of their expression levels. Plasmid DNA was directly extracted from collected HEK 293T cells and transformed into Escherichia coli for isolation and sequencing of inserts. Interestingly, 6 of 10 clones contained the scFv HA22 sequence, whereas 4 were scFv BL22 clones. Therefore, the single-pass enrichment of scFv HA22-displaying cells was 240-fold, although the difference in the affinity of the HA22 and the original BL22 (RFB4) is only $\approx 2$-fold. 
A

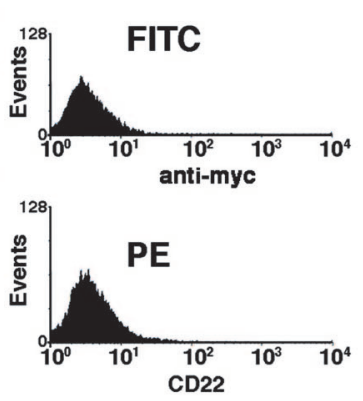

B
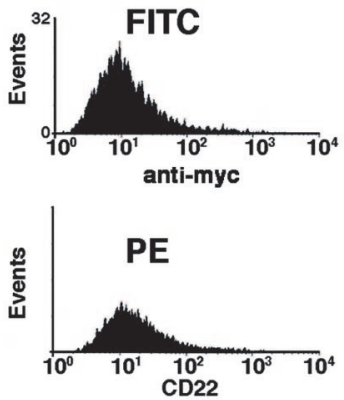
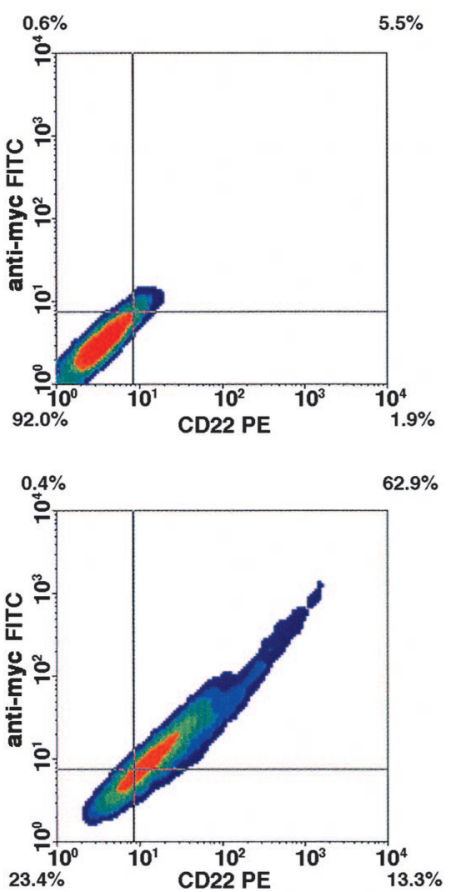

C
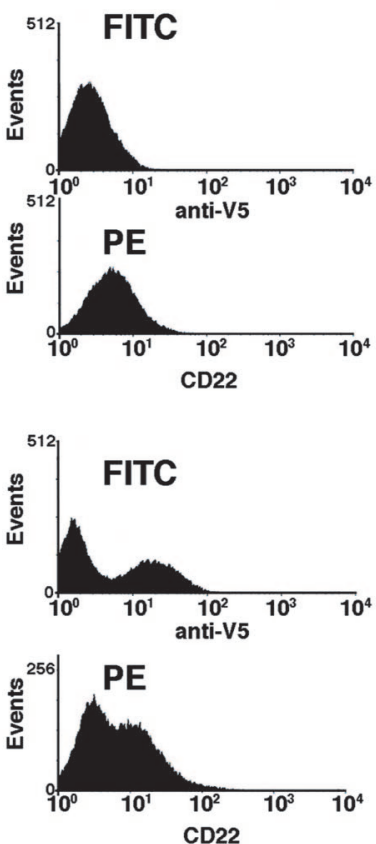

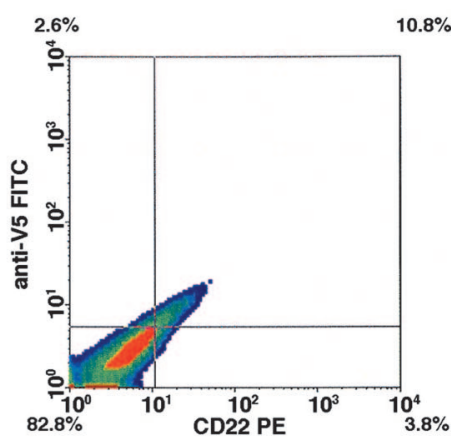

$12.5 \% \quad 30.0 \%$

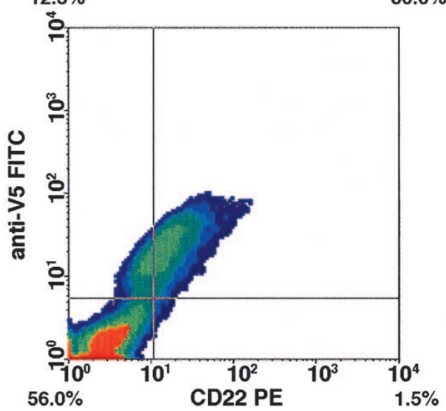

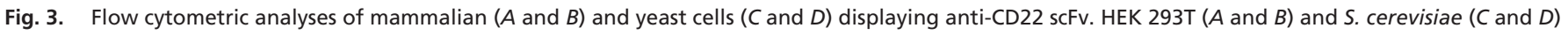

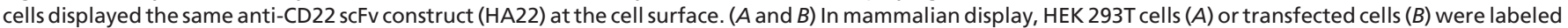

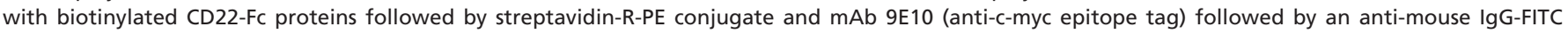

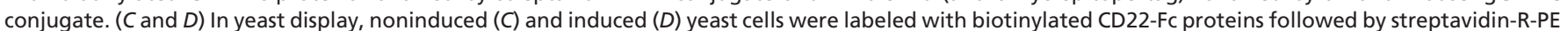

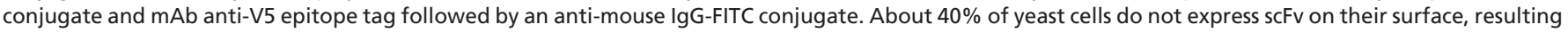
in two histogram peaks.

Construction of a HEK 293T Cell Surface Display Library for in Vitro Antibody Affinity Maturation. Finally, we asked whether this system could be suitable for isolating a high binder that could be used to make a more active immunotoxin or for other targeted therapies from a combinatory library. We constructed a HEK 293T cell surface-displayed combinatory library randomizing an intrinsic antibody hotspot. There are six complementary determining regions (CDRs), three on antibody heavy chain and three on light chain. CDRs are involved in antigen binding. Previous work has shown that a small number of DNA residues in CDRs called hotspots (30-33) can be used to increase antibody affinity $(9,10,34,35)$. Here we used the same strategy to select a hotspot (Gly-91-Asn-92) in light-chain CDR3 of HA22 to construct an anti-CD22 scFv library.

The scFv library was transduced into E. coli cells by electroporation. The library complexity of RFB4LibVL30/31 consisted of $>1 \times 10^{4}$ independent clones. Because a library of $1,024(32 \times$ 32) independent clones is needed to randomly mutate the hotspot sequence (GGTAAT) to NNSNNS, the size of the current library is 10 -fold larger than the one required. Sequence analysis of 10 independent clones demonstrated that the targeted hotspot residues were successfully randomized (data not shown). The library was then transfected into HEK 293T cells with Lipofectamine and the Fv expressed on the cell surface was fused to the PDGFR transmembrane domain (Fig. 1).

Isolation of Mutant scFv With a Higher Affinity from a HEK 293T Cell Surface Display Library. One transfection in a $100-\mathrm{mm}$ Petri dish can produce $\approx 10^{7}$ transfected HEK $293 \mathrm{~T}$ cells. Because the required library size is only $\approx 1,000$, the high transfection rate of one dish of HEK $293 \mathrm{~T}$ cells produced a library with $>10^{4}$ copies of each clone. Forty-eight hours after transfection, the mutant
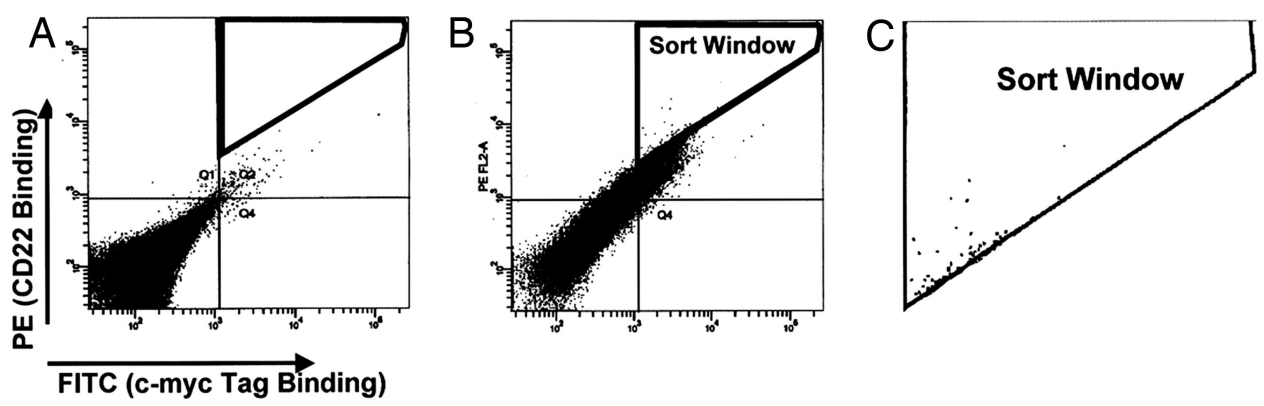

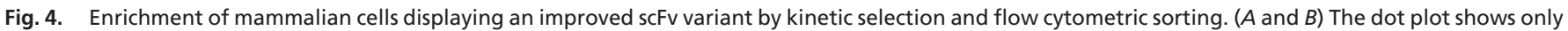

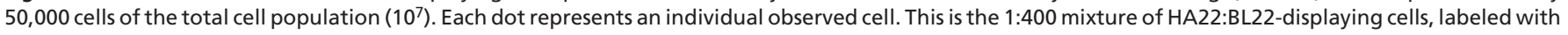

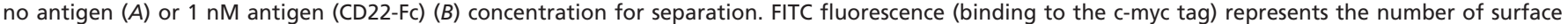

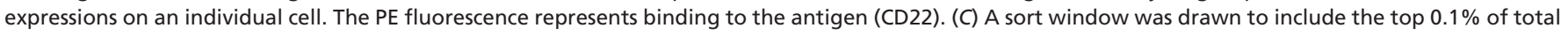
cells in terms of ratio of PE/FITC fluorescence (29). Cells that fell within the window were collected. 
A

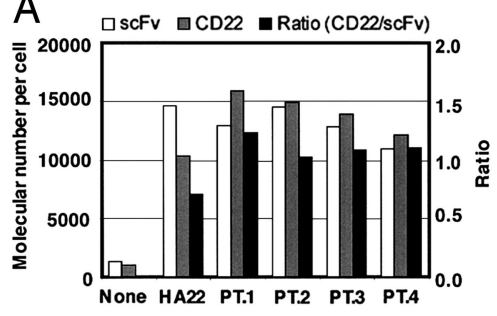

B

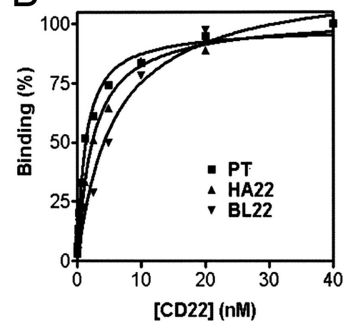

Table 1. The experimental procedure to make an Fv library for affinity maturation by mammalian cell surface display

\begin{tabular}{|c|c|c|}
\hline Step & Day & Procedure \\
\hline 1 & 1 & Prepare library plasmid DNA $(\approx 200 \mu \mathrm{g})$ \\
\hline 2 & 1 & $\begin{array}{l}\text { Grow one } 100-\mathrm{mm} \text { Petri dish of HEK } 293 \mathrm{~T} \text { cell to } \\
60 \% \text { confluence }\end{array}$ \\
\hline 3 & 2 & $\begin{array}{l}\text { The next day, transfect each dish with } 5 \mu \mathrm{g} \text { of } \\
\text { library DNA with Lipofectamine }\end{array}$ \\
\hline 4 & 4 & $\begin{array}{l}48 \mathrm{~h} \text { after transfection, harvest cells }\left(\approx 10^{7} \text { cells }\right. \\
\text { per dish) and perform flow cytometric sorting } \\
\text { of } 10^{7} \text { cells }(10,000-20,000 \text { cells per sec) }\end{array}$ \\
\hline 5 & 4 & $\begin{array}{l}\text { Collect } 0.1 \% \text { of cells }\left(\approx 10^{4}\right) \text { and recover plasmid } \\
\text { DNA from cells }\end{array}$ \\
\hline 6 & 4 & Transform the recovered plasmid DNA into $E$. col \\
\hline 7 & 5 & Pick E. coli colonies and grow overnight \\
\hline 8 & 6 & Isolate plasmids and run sequencing reaction \\
\hline 9 & 7 & $\begin{array}{l}\text { Obtain sequence and plan next selection if } \\
\text { necessary }\end{array}$ \\
\hline
\end{tabular}

Fig. 5. Interaction between CD22 and HEK 293T cell surface-displayed scFv. (A) Four individual clones (PT.1, PT.2, PT.3, and PT.4) of scFvs with the same mutation (PT) isolated from a single-pass enrichment were tested for the scFv expression (scFv) on HEK 293T cells with anti-myc mAb detected by FITC and for the binding to the antigen with CD22-Fc (CD22) detected by PE. Numbers of fluorocromes on a HEK 293 T cell were calculated by using calibration beads (see Materials and Methods). The numbers of CD22-bound scFvs and total scFvs and their ratios are shown with those of the original HA22 scFv on the surface of HEK 293 T cells. (B) Equilibrium binding titration curves to determine dissociation constants, $K_{\mathrm{D}}$. MFI (\%) of PE is plotted versus the various concentrations of biotinylated CD22-FC used to label surface-displayed BL22 (WT, $\left.K_{\mathrm{D}}=5.8 \mathrm{nM}, B_{\max }=453\right), \mathrm{HA} 22\left(K_{\mathrm{D}}=2.5 \mathrm{nM}, B_{\max }=293\right)$, or mutant $\mathrm{PT}\left(K_{\mathrm{D}}=\right.$ $\left.1.2 \mathrm{nM}, B_{\max }=275\right)$ antibody. Data are fit with a nonlinear least-squares regression, as described in Materials and Methods.

library of $1 \times 10^{7} \mathrm{HEK} 293 \mathrm{~T}$ cells was incubated with $0.2 \mathrm{nM}$ biotinylated CD22-Fc proteins at $25^{\circ} \mathrm{C}$ for $60 \mathrm{~min}$. The top $0.1 \%$ PE-positive cells (13,300 cells) were collected (Fig. 4C). Plasmids were recovered from selected HEK 293T cells and transformed into $E$. coli to recover plasmid DNA for sequencing. Typical recovery is at least one plasmid per cell. The Pro-91-Thr-92 (PT) mutation was found in 8 of 10 independent clones. The other 2 clones were Gly-91-Ala-92 and Val-91-Phe-92.

Binding Affinity Analysis of the New Mutant. Four individual clones each with the same PT mutation were analyzed for binding affinity. HEK 293T cell surface-displayed scFv HA22, and the PT mutants were incubated with $2 \mathrm{nM}$ biotinylated CD22-Fc, a concentration equivalent to the dissociation constant for CD22 of the starting scFv HA22 (10). The expression levels of the HA22 scFv and mutant scFvs were similar as measured by the mean fluorescence intensity (MFI) of FITC. In contrast, the CD22 binding measured as MFI of PE was remarkably higher in all of the PT mutants (Fig. 5A). The MFI of cells was converted to the number of molecules of fluorochrome per cell by using calibration beads. The transfected HEK 293 T cells have $\approx 15,000$ $\mathrm{scFv}$ molecules on the surface.

The CD22 binding affinity of each HEK 293T cell surfacedisplayed single-chain antibody was determined from equilibrium binding titration curves (29) obtained by incubating Fvexpressing cells in various concentrations of biotinylated CD22-Fc (see Materials and Methods). Fig. 5B shows results of two independent titrations of cell surface-displayed BL22, HA22, and PT. The equilibrium dissociation constant, $K_{\mathrm{D}}$, was fit by nonlinear least squares. The affinity of HEK $293 \mathrm{~T}$ cell surface-displayed PT mutant for CD22 $\left(K_{\mathrm{D}}=1.2 \mathrm{nM}\right)$ is $\approx 2$-fold higher than that for HA22 $\left(K_{\mathrm{D}}=2.5 \mathrm{nM}\right)$ and $\approx 5$-fold higher than that for WT BL22 $\left(K_{\mathrm{D}}=5.8 \mathrm{nM}\right)$. The HA22 and BL22 affinity values are consistent with those previously measured by flow cytometric analysis of Daudi cells using immunotoxin proteins (10).

\section{Discussion}

Here, we show that mammalian (HEK 293T) cells can be engineered to display a functional antibody Fv at the cell surface and be used to facilitate antibody affinity maturation. The results demonstrate that functional scFvs can be displayed on the cell

surface in a manner accessible for protein antigen recognition. Furthermore, the system is amenable to construction of a combinatorial library and high-throughput screening for affinity maturation by flow cytometry. We report that a mammalian cell surface display system can be used for in vitro antibody affinity maturation and serves as a proof of concept for an approach to antibody engineering. The scFv selected can be easily converted to whole IgG antibodies or immunotoxins, Fv-toxin chimeric proteins, for clinical use. The application of this display system in affinity maturation for antibodies, $\mathrm{T}$ cell receptors (36), and other biologically important molecules should be a significant alternative to existing technologies for affinity-based selections such as phage display, bacterial surface display, and yeast cell surface display.

Important features of this system are $(i)$ that it is compatible with other mammalian expression systems and (ii) that it is a rapid, robust, and relatively simple procedure. Starting with a Fv expression library it requires only 7 days to identify the DNA sequence of clones enriched by the selection and the DNA is ready for the next round of selection (Table 1). Microorganisms currently used in antibody engineering can have problems with protein folding, posttranslational modification, and codon usage (20). Serious problems often occur when antibody mutants selected in phage display are transferred into mammalian expression systems, which are widely used in the pharmaceutical industry to produce active therapeutic antibodies. Therefore, a mammalian expression system that can be used in antibody selection and affinity maturation would be very useful.

We have demonstrated the feasibility of this approach by fusing the single-chain antibody sequence at the amino terminus of the PDGFR transmembrane domain downstream of the signal sequence of the $\operatorname{Ig} \kappa$ chain. Other methods for expression on the cell surface could involve fusion to any natural receptor of choice, a glycosylphosphatidylinositol-anchored protein (24), or an artificial construct.

One important advantage of cell-based display over phage display is that we can monitor the expression level of each Fv on each cell by FACS and use the expression level to normalize the antibody-binding signal based on the number of Fvs on the cell surface. Expression systems typically exhibit a wide range of variation in the amount of protein displayed, and mutations in Fvs often produce mutant proteins with altered expression levels. These variations should not interfere with selections in a cell surface display system, making it possible to isolate better binders even with a slight difference in affinity. In a proof-ofconcept experiment, HEK 293 T cells expressing a rare mutant 
antibody were enriched 240 -fold by a single-pass cell sorting from a large excess of cells expressing WT antibodies with slightly lower affinity. The sort window was set to obtain the top $0.1 \%$ of cells with high CD22 binding normalized for Fv expression (Fig. 4). Under similar conditions, a single-pass enrichment of bacterial cells and yeast cells was 300- and 125-fold, respectively $(13,29)$.

One obvious challenge when using cell-based systems rather than in vitro-based ribosomal display is the limited library size that can be obtained because of restrictions in the transformation step. Phage display has the largest library size $\left(10^{9}\right)(19)$. The antibody library size in yeast is typically $10^{6}(37,38)$. Libraries of this size have been successfully used for many purposes. Such libraries may include hypothesis-driven (as described here) or semirandom mutagenesis for affinity maturation, human recombinant antibody libraries from patients, and hyperimmunized mouse spleen-derived recombinant libraries. In the present study, transient transfection of HEK 293 T cells in a $100-\mathrm{mm}$ dish produced $10^{7}$ individual clones. The same procedure can easily be expanded to 100 dishes in 1 day. The high-speed flow cytometric sorting machine (FACSVantage SE, BD Biosciences, San Jose, CA) has the capacity to screen $10^{9}$ cells in 1 day. Therefore, the library size of the mammalian display described here is comparable to phage and yeast display methods that are commonly used.

The high-affinity mutant described here may be useful in the treatment of patients with B cell malignancies. CD22 is a B cell antigen expressed in B cell lymphomas and leukemias and not expressed on stem cells or early in B cell development (39-41). We have shown that more than half of patients with drugresistant hairy cell leukemia can be brought into complete remission (42) by using the BL22 immunotoxin derived from anti-CD22 antibody (reviewed in ref. 43). The high-affinity mutant described here may expand the use of BL22 mutants in the treatment of patients with chronic lymphocytic leukemia, in which the cells have relatively small amounts of CD22 $(44,45)$.

\section{Materials and Methods}

Construction of Mammalian Cell Surface Display Library. Table 1 outlines the experimental procedure for making an Fv library for affinity maturation by mammalian cell surface display. We displayed mutant library of anti-CD22 scFv on HEK 293T cells. The surface display library was constructed by randomly mutagenizing a hotspot (Gly-91-Asn-92) in light-chain CDR3 of anti-CD22 HA22 scFv. HA22 scFv already contains mutations in the heavy-chain CDR3 hotspot of an anti-CD22 mAb RFB4 (35). The mutagenesis of HA22 scFv was performed as described (10). Degenerate oligomers with NNS were used (N randomizing with all four nucleotides, $\mathrm{S}$ introducing $\mathrm{C}$ or $\mathrm{G}$ ) in two-step overlap extension PCR: Dis_SfiIF, 5'-GGGGCCCAGCCGGCCATGGAAGTGCAGCTGGTG-3'; Dis_SacIIR，5'-CTGCCGCGGAGCTTTGATTTCCAG-3'; RFB4_VL91/92F，5' TTTTGCCAACAGNNSNNSACGCTTCCGTGG-3'; and RFB4_VL191/92R，5'-CCACGGAAGCGTSNNSNNCTGTTGGCAAAA-3'. Phagemid pCANTAB5E-scFv HA22 (clone G3) was used as a template. The PCR product was subcloned into SfiI and SacII sites downstream of the Ig $\kappa$ chain leader sequence of the pDisplay vector (Invitrogen). The vector encodes a myc epitope tag and a PDGFR transmembrane domain downstream of the $\mathrm{scFv}$. The ligation mix was used to transform electroporation-competent E. coli cells. Each transformation produced a cell surface display library containing $\approx 1 \times 10^{6}$ independent clones. The plasmid library, pDisplayRFB4VL91/92, contains a mutation targeting G91-N92 residues in light-chain CDR3 of the HA22 scFv. We also made plasmids pDisplay-BL22 (pMH112) and pDisplay-HA22 (pMH113) containing scFv BL22 (RFB4) and HA22, respectively, as controls.
Transfection. The scFv protein was expressed as a fusion to PDGFR transmembrane domain in transfected HEK 293T cells (10). Lipofectamine (Invitrogen) was used for transfections. Five micrograms of plasmid DNA was used for transfection in a $100-\mathrm{mm}$ Petri dish containing $10^{7}$ HEK 293 T cells per dish.

Confocal Fluorescence Microscopy. HEK 293T cells transfected with pMH113 were grown on cover slips. After 48 h, cells were washed with PBS and fixed in $1 \mathrm{ml}$ of $4 \%$ formaldehyde in PBS for $10 \mathrm{~min}$ at $25^{\circ} \mathrm{C}$. Cells were blocked with 5\% BSA in PBS for $30 \mathrm{~min}$ at $25^{\circ} \mathrm{C}$ and then incubated with $1 \mu \mathrm{g} / \mathrm{ml}$ of biotinylated CD22-Fc or CD30-Fc proteins and $20 \mu \mathrm{g} / \mathrm{ml}$ of mAb 9E10 (anti-c-myc) or IgG1 isotype control (Sigma) in PBS containing 5\% BSA and $0.1 \%$ sodium azide. After incubation for $90 \mathrm{~min}$ at $25^{\circ} \mathrm{C}$, cover slips were washed, incubated with Alexa Fluor-488-labeled goat anti-mouse IgG (1:500; Molecular Probes) and streptavidin-Alexa Fluor-594 conjugate (1:500; Molecular Probes) for $60 \mathrm{~min}$ at $25^{\circ} \mathrm{C}$, and washed. To visualize the nucleus, cells were stained with DAPI (Molecular Probes) for $5 \mathrm{~min}$ at $25^{\circ} \mathrm{C}$. Slides were analyzed in a Zeiss LSM 510 confocal microscope.

Flow Cytometric Analysis. In a typical experiment, $48 \mathrm{~h}$ after transfection, $1 \times 10^{6}$ cells $(\approx 10 \%$ of total HEK $293 \mathrm{~T}$ cells collected from a $100-\mathrm{mm}$ Petri dish) were used. The cells were incubated with $0.1-1 \mu \mathrm{g} / \mathrm{ml}$ of biotinylated CD22-Fc or CD30-Fc proteins and $20 \mu \mathrm{g} / \mathrm{ml}$ of mAb 9E10 (anti-c-myc) or IgG1 isotype control (Sigma) in $500 \mu \mathrm{l}$ of PBS containing 5\% BSA and $0.1 \%$ sodium azide (FACS buffer). After incubation for $1 \mathrm{~h}$ at $4^{\circ} \mathrm{C}$, the cells were washed with PBS and incubated on ice with 1:200 dilution of FITC-labeled goat anti-mouse FITC (AMI0408; BioSource International, Camarillo, CA) and streptavidin-R-PE conjugate (S866; Molecular Probes) for $1 \mathrm{~h}$. After washing, the cells were suspended in $0.5 \mathrm{ml}$ of FACS buffer, and the fluorescence associated with the live cells was measured with a FACS Calibur flow cytometer (BD Biosciences).

In some experiments (Fig. $5 A$ ), the MFI of cells was converted to the molecular number of fluorochrome (FITC, PE) on cells using Quantum FITC MESF (Bangs Laboratories, Carmel, IN) or QuantiBrite PE (BD Biosciences) calibration beads.

Kinetic Screen of Library by Sorting. Approximately $1 \times 10^{7} \mathrm{HEK}$ $293 \mathrm{~T}$ cells were incubated with $0.2 \mathrm{nM}$ biotinylated CD22-Fc proteins and $20 \mu \mathrm{g} / \mathrm{ml}$ of $9 \mathrm{E} 10 \mathrm{mAb}$ in PBS buffer at $25^{\circ} \mathrm{C}$ for $1 \mathrm{~h}$. Finally, the cells were rinsed with ice-cold PBS buffer and labeled with FITC-antimouse IgG and streptavidin-PE conjugate as described above. Samples were sorted on a FACSVantage SE (BD Biosciences) with a sort window as shown in Fig. 4. During a single-pass sorting, $1 \times 10^{7}$ HEK $293 \mathrm{~T}$ cells were examined and the window was set to collect $0.1 \%$ of the population.

Yeast Display. For a comparison between the mammalian cell surface display and yeast display, we expressed the HA22 scFv on yeast. The pYD1 vector (Invitrogen) and Saccharomyces cerevisiae strain EBY100 (14) was used for yeast cell surface display of anti-CD22 scFv HA22. The HA22 scFv was fused to the carboxyl terminus of Aga2p, and the fusion tethered to the yeast cell surface by disulfide bonding to the anchoring subunit Aga1p (Fig. 1). Each scFv contains a carboxyl-terminal V5 epitope tag. The pMH111 plasmid contains the HA22 scFv insert at the EcoRI and XhoI sites. Yeast cells were transformed with pMH111 by using the S.c. EasyComp transformation kit (Invitrogen), and Trp + transformants were selected. Yeast cells containing $\mathrm{scFv}$ surface display plasmids were grown in minimal SD-CAA medium $(20 \mathrm{~g} /$ liter dextrose $/ 6.7 \mathrm{~g} /$ liter yeast nitrogen base $/ 5 \mathrm{~g} /$ liter casamino acids/10.19 g/liter $\mathrm{Na}_{2} \mathrm{HPO}_{4} \cdot 7 \mathrm{H}_{2} \mathrm{O} / 8.56$ g/liter $\left.\mathrm{NaH}_{2} \mathrm{PO}_{4} \cdot \mathrm{H}_{2} \mathrm{O}\right)(14)$ overnight in a $30^{\circ} \mathrm{C}$ shaking incuba- 
tor. Cells were resuspended in galactose-containing medium SG-CAA (20 g/liter galactose $/ 6.7 \mathrm{~g} /$ liter yeast nitrogen base $/ 5$ g/liter casamino acids/10.19 g/liter $\mathrm{Na}_{2} \mathrm{HPO}_{4} \cdot 7 \mathrm{H}_{2} \mathrm{O} / 8.56 \mathrm{~g} /$ liter $\mathrm{NaH}_{2} \mathrm{PO}_{4} \cdot \mathrm{H}_{2} \mathrm{O}$ ) (14) to induce $\mathrm{scFv}$ display. Induction was carried out at $20^{\circ} \mathrm{C}$ for $48 \mathrm{~h}$.

In a typical protocol for flow cytometric analysis of yeast, cells were incubated on ice with $0.1-1 \mu \mathrm{g} / \mathrm{ml}$ of biotinylated CD22-Fc and mAb anti-V5 (10 $\mu \mathrm{g} / \mathrm{ml}$; Sigma) for $30 \mathrm{~min}$, followed by anti-mouse FITC conjugate (1:200) and streptavidin-PE conjugate (1:200) for $1 \mathrm{~h}$. FITC and PE fluorescence intensities were quantified with a FACalibur flow cytometer (Becton Dickinson).

Preparation and Purification of CD22. CD22-Fc fusion proteins were prepared as described (10). Biotinylation of CD22-Fc was performed according to the manufacturer's instruction (Pierce).

Determination of Affinity Constants $\left(\boldsymbol{K}_{\mathrm{D}}\right)$. Antibody affinity measurements were performed with flow cytometry following protocols as described $(16,29)$. Equilibrium constants and Scatchard plots were determined by using the Marquardt-Levenberg algorithm for nonlinear regression with PRISM software (version 3.0.2; GraphPad, San Diego).

Rescue of scFv Sequence. Plasmid DNA was isolated by using the QIAprep Spin Miniprep Kit following the manufacturer's instruction (Qiagen, Valencia, CA) from HEK 293T cells sorted on

1. Adams, G. P. \& Weiner, L. M. (2005) Nat. Biotechnol. 23, 1147-1157.

2. Foote, J. \& Eisen, H. N. (1995) Proc. Natl. Acad. Sci. USA 92, 1254-1256.

3. Batista, F. D. \& Neuberger, M. S. (1998) Immunity 8, 751-759.

4. Hoogenboom, H. R. (2005) Nat. Biotechnol. 23, 1105-1116.

5. Smith, G. P. (1985) Science 228, 1315-1317.

6. Winter, G., Griffiths, A. D., Hawkins, R. E. \& Hoogenboom, H. R. (1994) Annu. Rev. Immunol. 12, 433-455.

7. Low, N. M., Holliger, P. H. \& Winter, G. (1996) J. Mol. Biol. 260, 359-368.

8. de Bruin, R., Spelt, K., Mol, J., Koes, R. \& Quattrocchio, F. (1999) Nat. Biotechnol. 17, 397-399.

9. Chowdhury, P. S. \& Pastan, I. (1999) Nat. Biotechnol. 17, 568-572.

10. Ho, M., Kreitman, R. J., Onda, M. \& Pastan, I. (2005) J. Biol. Chem. 280, $607-617$.

11. Francisco, J. A., Campbell, R., Iverson, B. L. \& Georgiou, G. (1993) Proc. Natl. Acad. Sci. USA 90, 10444-10448.

12. Francisco, J. A. \& Georgiou, G. (1994) Ann. N. Y. Acad. Sci. 745, 372-382.

13. Georgiou, G., Stathopoulos, C., Daugherty, P. S., Nayak, A. R., Iverson, B. L. \& Curtiss, R., III (1997) Nat. Biotechnol. 15, 29-34.

14. Boder, E. T. \& Wittrup, K. D. (1997) Nat. Biotechnol. 15, 553-557.

15. Boder, E. T., Midelfort, K. S. \& Wittrup, K. D. (2000) Proc. Natl. Acad. Sci. USA 97, 10701-10705.

16. Feldhaus, M. J., Siegel, R. W., Opresko, L. K., Coleman, J. R., Feldhaus, J. M., Yeung, Y. A., Cochran, J. R., Heinzelman, P., Colby, D., Swers, J., et al. (2003) Nat. Biotechnol. 21, 163-170.

17. Hanes, J. \& Plückthun, A. (1997) Proc. Natl. Acad. Sci. USA 94, 4937-4942.

18. Hanes, J., Jermutus, L., Weber-Bornhauser, S., Bosshard, H. R. \& Plückthun, A. (1998) Proc. Natl. Acad. Sci. USA 95, 14130-14135.

19. Gold, L. (2001) Proc. Natl. Acad. Sci. USA 98, 4825-4826.

20. Urban, J. H., Schneider, R. M., Compte, M., Finger, C., Cichutek, K., Alvarez-Vallina, L. \& Buchholz, C. J. (2005) Nucleic Acids Res. 33, e35.

21. Schimmele, B., Gräfe, N. \& Plückthun, A. (2005) Protein Eng. Des. Sel. 18, 285-294.

22. Andersen, D. C. \& Reilly, D. E. (2004) Curr. Opin. Biotechnol. 15, 456-462.

23. Cumbers, S. J., Williams, G. T., Davies, S. L., Grenfell, R. L., Takeda, S., Batista, F. D., Sale, J. E. \& Neuberger, M. S. (2002) Nat. Biotechnol. 20, 1129-1134.

24. Wolkowicz, R., Jager, G. C. \& Nolan, G. P. (2005) J. Biol. Chem. 280, 15195-15201.

25. Gronwald, R. G., Grant, F. J., Haldeman, B. A., Hart, C. E., O'Hara, P. J., Hagen, F. S., Ross, R., Bowen-Pope, D. F. \& Murray, M. J. (1988) Proc. Natl. Acad. Sci. USA 85, 3435-3439. a FACSVantage SE (BD Biosciences). As few as 100 cells were enough for plasmid isolation. The plasmid DNA was transformed into TOP10F-competent $E$. coli (Invitrogen). Individual clones were used for sequencing and retransfection of HEK 293T cells. Alternatively, the plasmid DNAs recovered from HEK 293 T cells were used as templates for a PCR using the Tgo DNA polymerase with $3^{\prime}-5^{\prime}$ exonuclease proofreading activity (Roche Diagnostics).

Sequencing Analysis and Structural Modeling. The scFv fragments isolated from HEK 293T cell surface library were sequenced with primers Dis_SfiIF and Dis_SacIIR (National Cancer Institute DNA Sequencing Core Facility, Bethesda).

Both the heavy- and light-chains variable region sequences were numbered following the Kabat rule (46). Antibody Fv sequence analysis and structural modeling were performed as described (10).

We thank Dimiter Dimitrov (Protein Interactions at National Cancer Institute, Frederick, MD), Itai Benhar (Tel Aviv University, Tel Aviv), Lutz Jermutus (Cambridge Antibody Technology, Cambridge, U.K.), and Thomas Waldmann (National Cancer Institute) for critical reading of the manuscript; Barbara Taylor (National Cancer Institute Flow Cytometry Core Facility) and Susan Garfield (National Cancer Institute Confocal Microscopy Core Facility) for technical assistance; and Anna Mazzuca for editorial assistance. This research was supported by the Intramural Research Program of the National Institutes of Health, National Cancer Institute, Center for Cancer Research.

26. Santiago, C., Bjorling, E., Stehle, T. \& Casasnovas, J. M. (2002) J. Biol. Chem 277, 32294-32301.

27. Lee, J. Y., Zhao, L., Youn, H. S., Weatherill, A. R., Tapping, R., Feng, L., Lee, W. H., Fitzgerald, K. A. \& Hwang, D. H. (2004) J. Biol. Chem. 279, 1697116979

28. Zhu, D., Kepley, C. L., Zhang, K., Terada, T., Yamada, T. \& Saxon, A. (2005) Nat. Med. 11, 446-449.

29. VanAntwerp, J. J. \& Wittrup, K. D. (2000) Biotechnol. Prog. 16, 31-37.

30. Neuberger, M. S. \& Milstein, C. (1995) Curr. Opin. Immunol. 7, 248-254.

31. Neuberger, M. S., Ehrenstein, M. R., Klix, N., Jollcy, C. J., Rada, C. \& Milstein, C. (1998) Immunol. Rev. 162, 107-116.

32. Jolly, C. J., Wagner, S. D., Rada, C., Klix, N., Milstein, C. \& Neuberger, M. S. (1996) Semin. Immunol. 8, 159-168.

33. Rada, C., Ehrenstein, M. R., Neuberger, M. S. \& Milstein, C. (1998) Immunity 9, 135-141.

34. Beers, R., Chowdhury, P., Bigner, D. \& Pastan, I. (2000) Clin. Cancer Res. 6, 2835-2843.

35. Salvatore, G., Beers, R., Margulies, I., Kreitman, R. J. \& Pastan, I. (2002) Clin. Cancer Res. 8, 995-1002.

36. Holler, P. D., Holman, P. O., Shusta, E. V., O'Herrin, S., Wittrup, K. D. \& Kranz, D. M. (2000) Proc. Natl. Acad. Sci. USA 97, 5387-5392.

37. Blaise, L., Wehnert, A., Steukers, M. P., van den Beucken, T., Hoogenboom, H. R. \& Hufton, S. E. (2004) Gene 342, 211-218.

38. Jin, M., Song, G., Carman, C. V., Kim, Y. S., Astrof, N. S., Shimaoka, M., Wittrup, D. K. \& Springer, T. A. (2006) Proc. Natl. Acad. Sci. USA 103, 5758-5763.

39. Tedder, T. F., Tuscano, J., Sato, S. \& Kehrl, J. H. (1997) Annu. Rev. Immunol. 5, 481-504.

40. Tuscano, J. M., O’Donnell, R. T., Miers, L. A., Kroger, L. A., Kukis, D. L., Lamborn, K. R., Tedder, T. F. \& DeNardo, G. L. (2003) Blood 101, 3641-3647.

41. Tedder, T. F., Poe, J. C. \& Haas, K. M. (2005) Adv. Immunol. 88, 1-50.

42. Kreitman, R. J., Wilson, W. H., Bergeron, K., Raggio, M., Stetler-Stevenson, M., FitzGerald, D. J. \& Pastan, I. (2001) N. Engl. J. Med. 345, 241-247.

43. Pastan, I. (2003) Cancer Immunol. Immunother. 52, 338-341.

44. Kreitman, R. J., Margulies, I., Stetler-Stevenson, M., Wang, Q. C., FitzGerald, D. J. P. \& Pastan, I. (2000) Clin. Cancer Res. 6, 1476-1487.

45. Rossmann, E. D., Lundin, J., Lenkei, R., Mellstedt, H. \& Osterborg, A. (2001) Hematol. J. 2, 300-306.

46. Kabat, E. A., Wu, T. T., Reid-Miller, M., Perry, H. M., Gottesman, K. S. \& Foeller, C. (1991) Sequences of Proteins of Immunological Interest (National Institutes of Health, Bethesda), 5th Ed. 